\title{
Photometric evidence for two-temperature photospheric inhomogeneities on magnetically active K dwarf stars (Research Note)
}

\author{
S. Messina ${ }^{1}$, G. Cutispoto ${ }^{1}$, E. F. Guinan ${ }^{2}$, A. F. Lanza ${ }^{1}$, and M. Rodonò ${ }^{1,3}$ \\ 1 INAF - Catania Astrophysical Observatory, via S. Sofia 78, 95123 Catania, Italy \\ e-mail: [sme; gcutispoto;nlanza]@oact.inaf.it \\ 2 Dept. of Astronomy and Astrophysics, Villanova University, Villanova 19085, PA, USA \\ e-mail: edward.guinan@villanova.edu \\ 3 Dept. of Physics and Astronomy, Catania University, via S. Sofia 78, 95123 Catania, Italy \\ e-mail: mrodono@oact.inaf.it
}

Received 7 August 2005 / Accepted 29 September 2005

\section{ABSTRACT}

We derive the properties of spotted regions in late-type active stars from $V$ - and $B$-band photometric data. Specifically, we compare the amplitudes of spot-induced light and color variations with model amplitudes, which depend on the area, temperature and distribution of spotted regions over the stellar surface. Our analysis is applied to three well-known chromospherically active K dwarf stars: DX Leonis, AB Doradus and LQ Hydrae, and indicates that all three stars show significant variations of the average spot temperature from epoch to epoch. The observed temporal variations of the starspot temperatures are best explained as arising from the contemporary presence on the stellar photosphere of two-component (two-temperature) active regions. These consist of either dark spots and hot faculae and/or dark umbrae and penumbrae and the variations over time arise from change of their areal ratio.

Key words. stars: late-type - stars: starspots - stars: activity - stars: individual: DX Leonis - stars: individual: AB Doradus stars: individual: LQ Hydrae

\section{Introduction}

The magnetic fields in the photospheres of late-type stars arise from the hydro-magnetic processes likely operating in the overshoot layer near the base of their external convective zones. The properties of photospheric magnetized plasmas, notably temperature, area and distribution over the stellar photosphere, depend also on the magneto-convective processes occurring in the surface layers. However, the measurement of these properties turns out to be relevant to constrain the magneto-convection models and, even if to a much lesser extent, some basic assumptions of the dynamo models.

The light curve inversion is the method which has been widely used to extract information on the properties of active regions from wide- and intermediate-passband photometric data and to re-construct maps of the stellar photosphere. Regularization criteria, such as the Maximum Entropy method and the Tikhonov criterion, allow us to overcome the nonuniqueness of solutions. Much work has been done to model dark spots, while much less effort has been payed to investigate bright photospheric faculae, whose presence in active stars is still debated. In fact, some authors have found that for the less active stars the variability at the optical wavelengths is significantly influenced by faculae, if not quite dominated, e.g. Mirtorabi et al. (2003), Radick et al. (1990, 1998), while for the most active stars the effect of starspots dominates and faculae are not required to obtain quite satisfactory light curves models (e.g. Henry et al. 1995; Lanza et al. 1998). Other authors have recently shown that also in more active stars faculae are needed either to account for their UV flux excess with respect to inactive stars (e.g. Amado 2003) or to model the $B-V$ color blueing when they get fainter, as proposed, e.g., by Aarum Ulvås \& Engvold (2003) and Aarum Ulvås \& Henry (2005) in the case of UX Ari, V711 Tau and RS CVn.

When single-band photometric data are modelled, the temperature of starspots is assumed and usually kept constant versus time on the basis of statistical values retrieved from the literature or obtained by means of multi-band data (such as UBVRI or Strömgren uvby photometry) or from spectroscopic methods such as the Line Depth Ratio (Gray 1994; Catalano et al. 2002). Two-band photometric data, at least, and notably those from NIR passbands, are needed to derive information about spot temperature. Nonetheless, $B$ - and $V$-band photometry permits to investigate the spot temperature, with the relevant advantage of extending such an investigation to long-term sequences of photometric data. In fact, the $B$ and $V$ bands of the 
Table 1. Sources of photometric data.

\begin{tabular}{|c|c|}
\hline $\begin{array}{c}\text { Mean epoch } \\
\text { (yr) }\end{array}$ & Source \\
\hline \multicolumn{2}{|r|}{ AB Dor } \\
\hline 1984.97 & Jetsu et al. (1990) \\
\hline 1986.84 & Kubiak (1985) \\
\hline 1987.93 & Cutispoto (unpublished) \\
\hline 1989.12 & Kürster et al. (1994) \\
\hline 1989.96 & Cutispoto (1995) \\
\hline \multicolumn{2}{|r|}{ LQ Hya } \\
\hline 1991.22 & Cutispoto (1998a) \\
\hline 1992.14 & Cutispoto (1998b) \\
\hline & Jetsu (1993) \\
\hline 1993.02 & Cutispoto et al. (2001) \\
\hline 2000.96 & Covino et al. (2002) \\
\hline 2001.13 & Berdyugina et al. (2002) \\
\hline & Covino et al. (2002) \\
\hline \multicolumn{2}{|r|}{ DX Leo } \\
\hline 1996.22 & Messina \& Guinan (2002) \\
\hline 1996.32 & “ \\
\hline 1996.88 & $"$ \\
\hline 1996.85 & “ \\
\hline 1997.05 & $"$ \\
\hline
\end{tabular}

Johnson's system have been used since photometric monitorings of late-type stars have begun.

In this paper we explore the extraction of information on the temperatures of either dark spots and bright faculae from $B$ and $V$-band data. We have focused our study on three K-type dwarf stars: AB Dor, LQ Hya and DX Leo which have similar global physical parameters, but different rotation rates and, therefore, different magnetic activity levels. AB Dor is the most active star of the small sample. We briefly describe the photometric data in Sect. 2, our modeling approach in Sect. 3, while results and conclusions are presented in Sect. 4.

\section{Observations}

AB Dor (HD 36705, $P_{\text {rot }}=0.51479$ ) is a very active young K0 dwarf star showing flux rotational modulation with a light amplitude up to $\Delta V=0.26 \mathrm{mag}$, a starspot cycle of about $P_{\text {cyc }} \sim 18 \mathrm{yr}$ and evidence for a solar-like pattern of surface differential rotation with amplitude $\Delta \Omega / \Omega \simeq 0.4 \%$ (Järvinen et al. 2005; Collier Cameron \& Donati 2002). For this study we selected five mean epochs when the star was observed simultaneously in the $V$ and $B$ passbands and the observations allowed us to secure light curves with a good rotational phase coverage. The photometric data were taken from a number of sources as listed in Table 1. Jetsu's data were transformed from the uvby system into the $U B V$ standard system according to Olsen (1983). In Fig. 1 (left-hand panels) we plot the light curves, where rotational phases were computed using the ephemeris HJD $=2444296.575+0.51479 \times E$ (Innis et al. 1988). As shown, in this figure (right-hand panels) we plot the color- $V$ mag relationships analysed in this paper, where $(B-V)_{0}$ is the bluest color from the plotted linear best fit and $V_{0}$ is the observed brightest magnitude of each light curve, respectively.
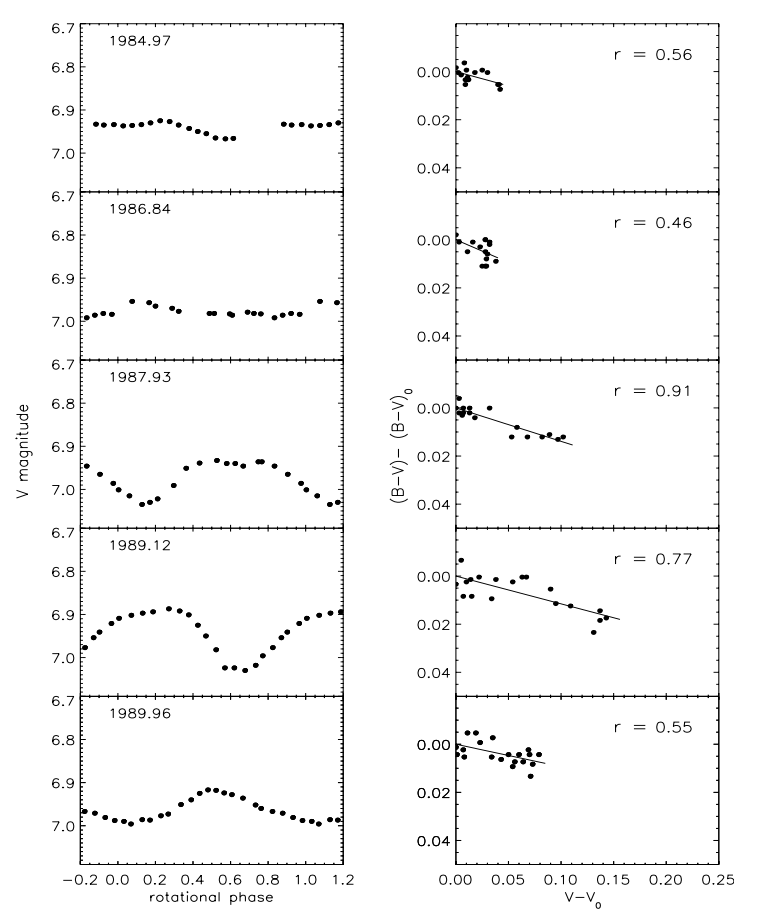

Fig. 1. Left hand panels: a selection of light curves of AB Dor. $V$ magnitude vs. rotational phase. Rotational phases were computed using the ephemeris HJD $=2444296.575+0.51479 \times$ E. Right hand panels: $B-V$ color vs. $V$ magnitude variations. Solid lines are linear best fits to the data. $(B-V)_{0}$ and $V_{0}$ are the bluest color fit and the observed brightest magnitude. The correlation coefficients $(r)$ between $(B-V)-(B-V)_{0}$ and $\left(V-V_{0}\right)$ are indicated in each plot, respectively.

LQ Hya (HD 82558, $P_{\text {rot }}=1$ d.6042) is a well-known K2V active star with an amplitude of the flux rotational modulation up to $\Delta V=0.21 \mathrm{mag}$, multiple starspot cycles and evidence for a solar-like pattern of surface differential rotation with relative amplitude $\Delta \Omega / \Omega \geq 1.3 \%$ (Messina \& Guinan 2003). The light curves and the color-V mag relationships of LQ Hya are plotted in Fig. 2, where rotational phases are computed using the initial epoch HJD = 2448270.0 and the corresponding seasonal rotation period, as listed in Table 8 of Messina \& Guinan (2003). The photometric data were taken from several sources as listed in Table 1.

DX Leo (HD 82443, $\left.P_{\text {rot }}=5.424\right)$ is also a very active K0 dwarf star showing flux rotational modulation with an amplitude up to $\Delta V=0.11 \mathrm{mag}$, a prominent starspot cycle of $P_{\text {cyc }} \simeq 3.21 \mathrm{yr}$ and some evidence for an antisolar pattern of surface differential rotation with relative amplitude $\Delta \Omega / \Omega \geq 2 \%$ (Messina \& Guinan 2003). From the complete dataset of $V$-band photometry presented by Messina \& Guinan (2002), we focused our analysis on a selection of five mean epochs when $B$ - and $V$-band observations were contemporaneously collected. In Fig. 3 we plot the DX Leo light curves, where rotational phases are computed using the initial epoch $\mathrm{HJD}=2447570.0$ and the corresponding seasonal rotation period, as listed in Table 7 of Messina \& Guinan (2003), and the color- $\mathrm{V}$ mag relationships.

The photometric observations generally have rotational phases not equally spaced and present some dispersion around 

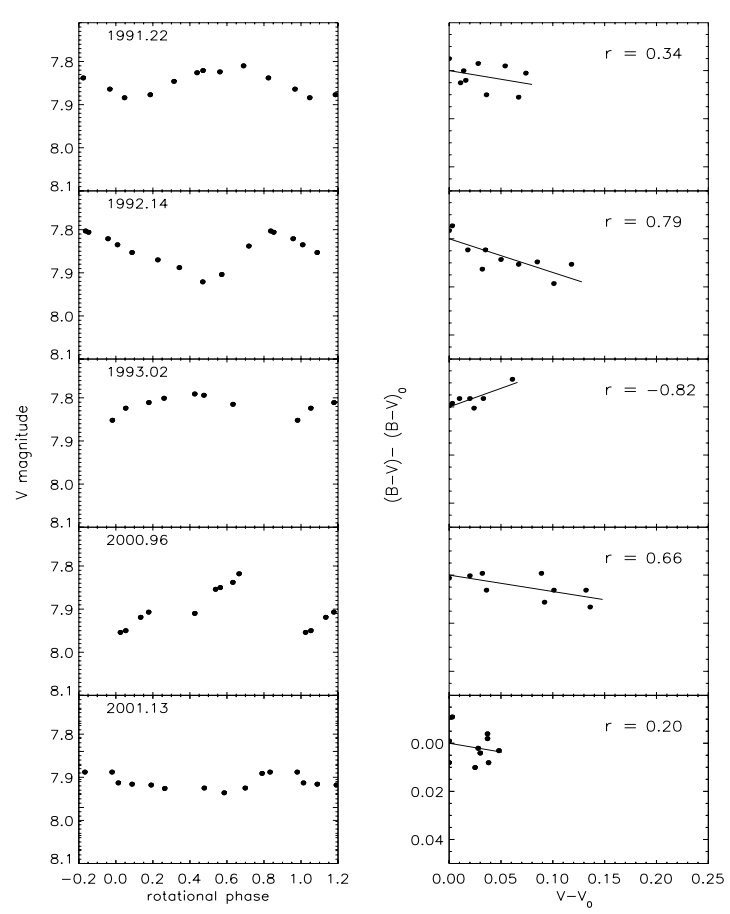

Fig. 2. Same as Fig. 1 but for LQ Hya.

their mean value at any given phase. Most of this scatter is attributed to the photometric precision (which is of the order of 0.010 and 0.008 mag for the $B$ and $V$ magnitudes, respectively) rather than to an intrinsic evolution of spotted regions. In fact, the light curves were selected in such a way to cover an interval of time during which the optical flux showed regular modulations suggesting quasi-stable spot patterns. Therefore, we decided to average the $V$ magnitudes and $B-V$ colors over 0.05 rotational phase bins and then to compute linear fits by giving the proper statistical weight to each average point. The bluest $(B-V)$ color fit and the brightest $V$ magnitude, labeled as $(B-V)_{0}$ and $V_{0}$, respectively, were assumed as the zero points in our analysis. In this analysis the reddest $B-V$ fit value and the corresponding faintest $V$ magnitude were used to compute the amplitude of color and magnitude variations to be modeled. As shown in the right-hand plots of Figs. 1-3, we found that linear trends exist in the relation of $(B-V)-(B-V)_{0}$ vs. $\left(V-V_{0}\right)$ with a slope changing from epoch to epoch. Also the linear Pearson correlation coefficient $(r)$ was found to vary over time. Such results support the idea that a common physical mechanism, which we further investigate, is operating in such a way as to affect both the stellar $V$ magnitude and the $B-V$ color with a similar time dependence.

\section{The model}

$V$ magnitude and $B-V$ color variations at any given mean epoch arise from the spotted regions which, being asymmetrically distributed along the stellar longitude, show different projected areas to the observer as the star rotates. Our aim is to model the amplitudes of such variations to derive information on those spotted regions, notably their areas and temperatures. Synthetic $V$ magnitude and $B-V$ color curve amplitudes were
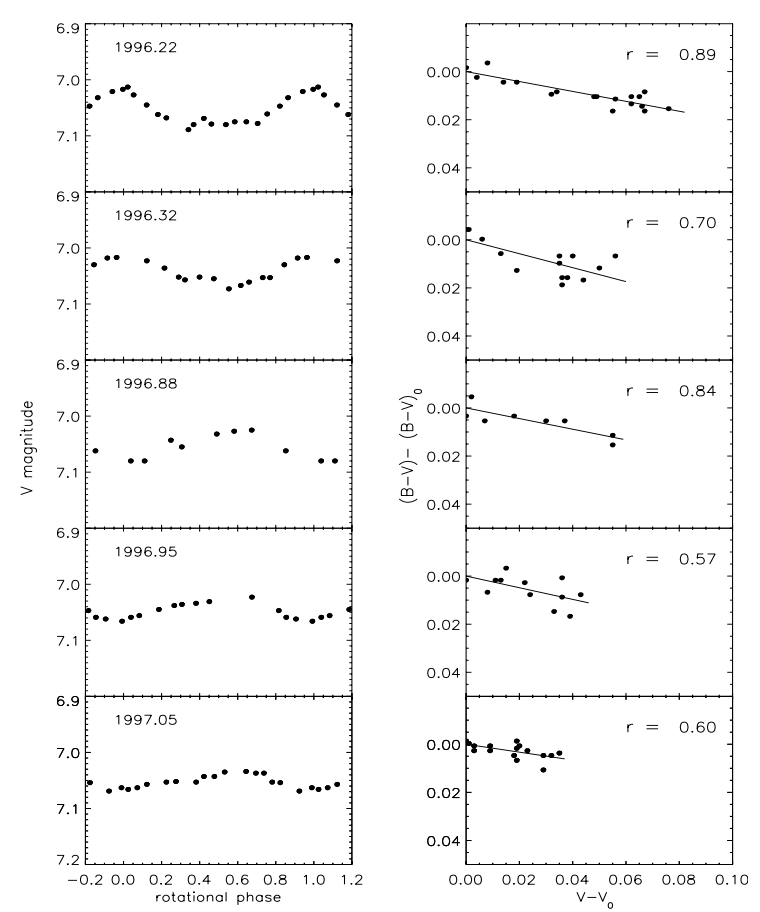

Fig. 3. Same as Fig. 1 but for DX Leo.

obtained using the approach proposed by Dorren (1987). We point out that we modeled the light and color curve amplitudes and not their shapes. Therefore, we considered the difference of magnitude and color between the unspotted (or the least spotted) and the most spotted hemisphere in the $B$ and $V$ bands. We expect that a single circular spot, when re-producing the observed light and color curve amplitudes, is a satisfactory approximation of any other differently shaped and asymmetrically distributed spotted region. The NextGen model atmosphere grid of Hauschildt et al. (1999) was adopted to compute synthetic stellar fluxes from the unspotted photosphere, and from the spot components unevenly and evenly distributed along stellar longitude, the latter affecting the mean magnitude. Synthetic $V$-band magnitude and $B-V$ colors were computed by convolving theoretical stellar fluxes with the passbands of the $U B V$ system (Johnson \& Morgan 1953) as tabulated in Buser (1978) and Buser \& Kurucz (1978). Limb-darkening coefficients from Diaz-Cordoves et al. (1995) were assigned to the unspotted and spotted photospheres according to their different temperatures. The input parameters of the model, i.e., the stellar unperturbed effective temperature $\left(T_{\text {eff }}\right)$, the surface gravity $(\log g)$ and the inclination of the stellar rotational axis $(i)$ were determined independently of the present dataset.

For AB Dor we adopted a value of $T_{\text {eff }}=5200 \mathrm{~K}$ and $\log g=4.5 \mathrm{~cm} \mathrm{~s}^{-2}$. The inclination was assumed to be $i=$ $60^{\circ}$ as discussed by Donati \& Collier Cameron (1997). $V=$ +6.756 mag and $B-V=+0.807$ mag were assumed as the unspotted magnitude and color, respectively (Järvinen et al. 2005).

For LQ Hya we adopted a value of $T_{\text {eff }}=5070 \mathrm{~K}, \log g=$ $4.5 \mathrm{~cm} \mathrm{~s}^{-2}$ and $i=65^{\circ}$ as discussed by Kövári et al. (2004). $V=+7.75 \mathrm{mag}$ and $B-V=+0.89 \mathrm{mag}$ were assumed as the unspotted magnitude and color, respectively (Jetsu 1993). 

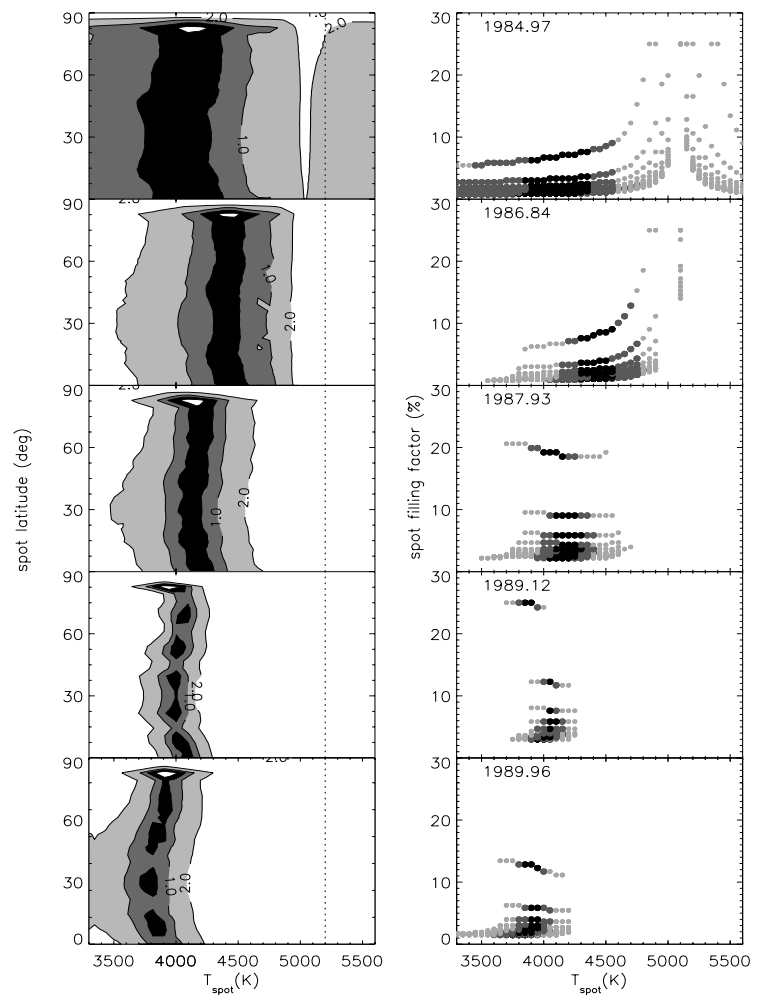

Fig. 4. Left hand panels: isocontour plots of the residuals of the fits to the observed $V$ vs. $B-V$ variation amplitudes of AB Dor in latitudetemperature planes. The three gray levels, from dark to light gray, indicate $\chi^{2}$ in the ranges $0-0.5,0.5-1$ and $1-2$, respectively. Right hand panels: distribution of spot filling factor (\%) vs. spot temperature. Gray levels have the same meaning than in left-hand panels. Each dotted-like line of filling factor values corresponds, from bottom to top, to the model solutions as derived for the spot latitudes from $0^{\circ}$ (bottom) to $90^{\circ}$ (top) in increments of $5^{\circ}$.

For DX Leo we adopted a value of $T_{\text {eff }}=5372 \mathrm{~K}$ as spectroscopically derived by Gray et al. (2003) on the basis of the spectral type and a value of $\log g=4.52 \mathrm{~cm} \mathrm{~s}^{-2}$. The inclination $i=50^{\circ}$ was derived from the mean rotational period $P_{\text {rot }}=$ 5.424 , the measured $v \sin i=6.0 \mathrm{~km} \mathrm{~s}^{-1}$ (see Messina et al. 1999) and an estimated stellar radius $R=0.83 R_{\odot}$, appropriate for its spectral type. $V=+7.01 \mathrm{mag}$ and $B-V=+0.75 \mathrm{mag}$ were assumed as the unspotted magnitude and color, respectively (Messina \& Guinan 2002).

Synthetic $V$ and $B-V$ curve amplitudes were generated for a grid of values of the spot temperature in the range $3000-5600 \mathrm{~K}$ with a step of $50 \mathrm{~K}$, of spot radius in the range $0-60^{\circ}$ with a step of $1^{\circ}$, and of spot latitude in the range $0-90^{\circ}$ with a step of $5^{\circ}$. The linear limb-darkening coefficients of the spotted region were varied according to the running spot temperature.

\section{Discussion and conclusions}

As expected from the use of photometric data alone, the model solutions are not unique. However, the signatures of active regions in the $B$ and $V$ photometric data are sufficient to allow the solutions to cluster around a finite range of values of the spot temperature at any given mean epoch. In Figs. 4-6 we plot the
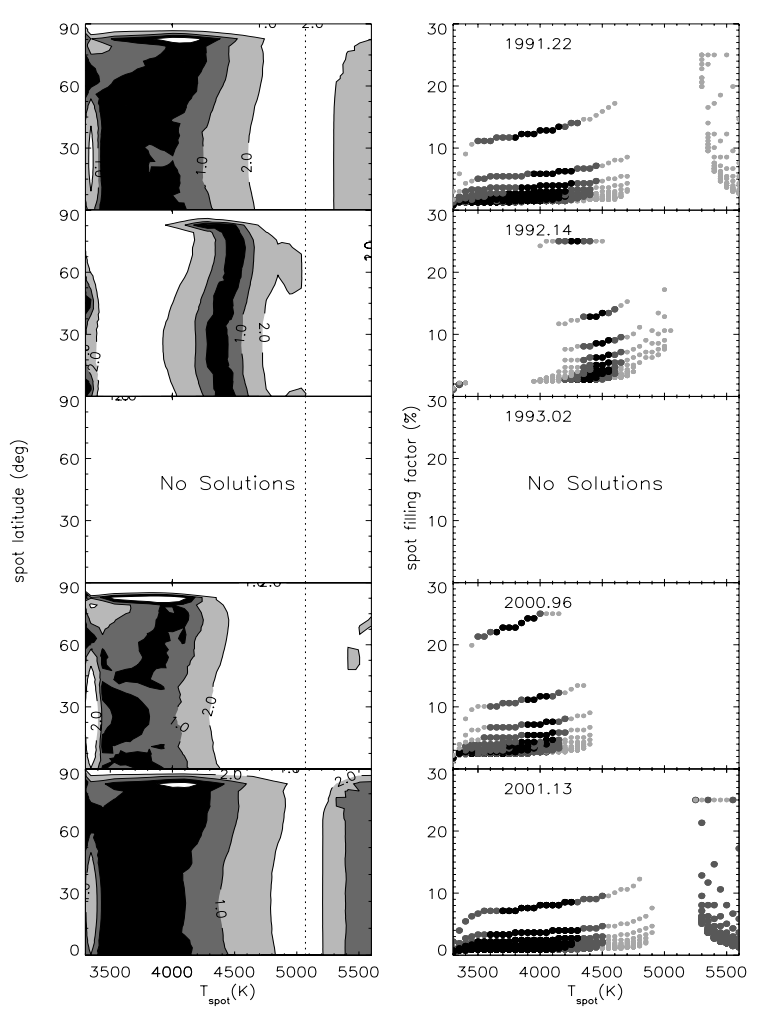

Fig. 5. Same as Fig. 4 but for LQ Hya.

distribution of the $\chi^{2}$ of the fit to the observed $V$ and $B-V$ variation amplitudes. At any given mean epoch, a proper weight was given to the values of the amplitudes to be fitted. We considered all the solutions in the $\chi^{2}$ range from 0 to 2 . Different gray levels of the residual isocontour plots represent the best fit solutions in the $\chi^{2}$ ranges $0-0.5$ (black), 0.5-1.0, 1.0-2.0 (light gray). The spot filling factor values, which in Figs. 4-6 are computed with respect to the whole stellar surface, tend to increase as far as the spot temperature and mean spot latitude increase. In fact, the lower brightness contrast between warmer spots and unperturbed photosphere, and the lower spot projected area of higher latitude spots require additional spotted area to produce the same variation amplitudes. All three stars show significant variations of the average spot temperature from epoch to epoch. The most reasonable explanation for such variations is that we are measuring the average temperature of two-component (two-temperature) active regions which are asymmetrically distributed in longitude. Such regions may be composed of either dark spots and bright faculae and/or cool and dark umbrae surrounded by warmer penumbrae, as in the solar case. The variations of the average starspot temperature may also originate from the changing spot/plages as well as umbra/penumbra areal ratio.

A temporal change of the umbra/photosphere temperature ratio may also be responsible for the observed behaviour. For the Sun the umbral temperature, as measured at near infrared wavelengths, has been found to change along the phase of the solar cycle. It has been found that the umbrae near the maximum are warmer by about $20 \%$ than near the cycle minimum (Maltby 1992). In the case of AB Dor and DX Leo the variation of the average spot temperature is of about $10 \%$, increasing up 

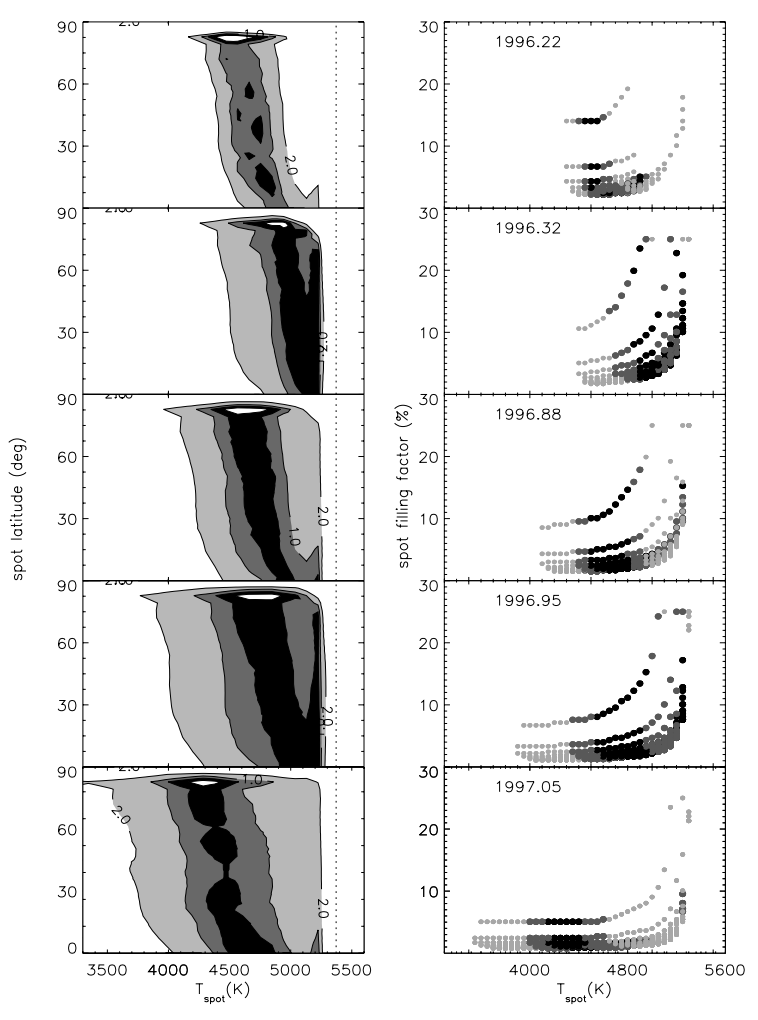

Fig. 6. Same as Fig. 4 but for DX Leo.

to $15 \%$ in the case of LQ Hya. However, a much more extended time series of photometric data is needed to investigate how the amplitude of the spot temperature variation and its correlation with the spot cycle phase behave in comparison to the solar case.

It is interesting to note that for LQ Hya, at the mean epoch 1993.02 the star tended to become bluer when it was fainter. In this case our two-component model was unable to find any satisfactory solution, at least in the range of parameter values we investigated. However, it is possible that the observed color variation may have been induced by a flare event occurred near the light curve minimum rather than by starspots. In fact, the slope of the $(B-V)-(B-V)_{0}$ vs. $\left(V-V_{0}\right)$ relation has negative sign because of only one data point corresponding to the light curve minimum. As show in Fig. 30 of Cutispoto et al. (2001) from where these data are taken, the "blueing" of the $U-B$ color, and to a lesser extent of the $B-V$ color, though no flux increase is detected at all in the $V$ band, may be consistent with the starting or ending phases of a stellar flare. In the case of a flare, the model assumption that magnitude and color variations are spot-induced are no longer valid, thus explaining why the model fails to find any acceptable solution.

A very important result we derive from the present analysis is that a two-component structure of active regions is required to fit the observed light and color variation amplitudes in the selected sample of active K dwarfs. Indeed, photometric evidence for two-temperature photospheric inhomogeneities is obtained for the first time in the case of active $\mathrm{K}$ dwarf stars. The highertemperature component may possibly be cooler than the unperturbed photosphere representing a penumbral-like component, or may be hotter, representing a facular-like component, as observed in solar active regions. Another important result is that the $B$ and $V$ photometric data contain significant information on the properties of active regions, which can be extracted following the approach outlined above.

Acknowledgements. This work was supported by the Italian MIUR (Ministero dell'Istruzione, Università e Ricerca) and by the USA National Science Foundation for Research. We thank the Referee Dr. V. Aarum Ulvås for careful reading of the manuscript and valuable comments.

\section{References}

Aarum Ulvås, V., \& Engvold, O. 2003, A\&A, 399, L11

Aarum Ulvås, V., \& Henry, G. W. 2005, AN, 326, 292

Amado, P. J. 2003, A\&A, 404, 631

Berdyugina, S. V., Pelt, J., \& Tuominen, I. 2002, A\&A, 394, 505

Buser, R. 1978, A\&A, 62, 411

Buser, R., \& Kurucz, R. L. 1978, A\&A, 70, 555

Catalano, S., Biazzo, K., Frasca, A., \& Marilli, E. 2002, A\&A, 394, 1009

Collier Cameron, A., \& Donati, J. F-. 2002, MNRAS, 329, 23

Covino, S., Tagliaferri, G., Bertone, E., et al. 2002, in Stellar Coronae in the Chandra and XMM-Newton Era, ed. F. Favata, \& J. J. Drake, ASP Conf. Ser., 277, 357

Cutispoto, G. 1995, A\&AS, 111, 507

Cutispoto, G. 1998a, A\&AS, 127, 207

Cutispoto, G. 1998b, A\&AS, 131, 321

Cutispoto, G., Messina, S., \& Rodonò, M. 2001, A\&A, 367, 910

Diaz-Cordoves, J., Claret, A., \& Gimenez, A. 1995, A\&A, 110, 329

Donati, J.-F., \& Collier Cameron, A. 1997, MNRAS, 291, 1

Dorren, J. D. 1987, ApJ, 320, 756

Gray, D. F. 1994, PASP, 106, 1248

Gray, R. O., Corbally, C. J., Garrison, R. F., McFadden, M. T., \& Robinson, P. E. 2003, AJ, 126, 2048

Hauschildt, P. H., Allard, F., \& Baron, E. 1999, ApJ, 512, 377

Henry, G. W., Eaton, J. A., Hamer, J., \& Hall, D. S. 1995, ApJS, 97, 513

Innis, J. L., Thompson, K., Coated, D. W., \& Lloyd Evans, T. 1988, MNRAS, 235, 1411

Järvinen, S. P., Berdyugina, S. V., Tuominen, I., Cutispoto, G., \& Bos, M. 2005, A\&A, 432, 657

Jetsu, L. 1993, A\&A, 276, 345

Jetsu, L., Vilhu, O., \& La Dous, C. 1990, A\&AS, 85, 1127

Johnson, H. L., \& Morgan, W. W. 1953, ApJ, 117, 313

Kövári, Z., Strassmeier, K. G., Granzer, T., et al. 2004, A\&A, 417, 1047

Kubiak, M. 1985, AcA, 35, 369

Kürster, M., Schmitt, J. H. M. M., \& Cutispoto, G. 1994, A\&A, 289, 899

Lanza, A. F., Catalano, S., Cutispoto, G., Pagano, I., \& Rodonò, M. 1998, A\&A, 332, 541

Maltby, P. 1992, in Sunspots: Theory and Observations, ed. J. H. Thomas, \& N. O. Weiss (Dordrecht: Kluwer Ac. Pub.), 103

Messina, S., \& Guinan, E. F. 2002, A\&A, 393, 225

Messina, S., \& Guinan, E. F. 2003, A\&A, 409, 1017

Messina, S., Guinan, E. F., Lanza, A. F., \& Ambruster, C. 1999, A\&A, 347,249

Mirtorabi, M. T., Wasatonic, R., \& Guinan, E. F. 2003, AJ, 125, 3265

Olsen, E. H. 1983, A\&AS, 54, 550

Radick, R. R., Lockwood, G. W., \& Baliunas, S. L. 1990, Science, 247, 39

Radick, R. R., Lockwood, G. W., Skiff, B. A., \& Baliunas, S. L. 1998, ApJS, 118, 239 